OPEN ACCESS

Edited by:

Heike Wulff,

University of California, Davis,

United States

Reviewed by:

Pallab Bhattacharya,

National Institute of Pharmaceutical

Education and Research

Ahmedabad, India

Martha Eaton O'Donnell,

University of California, Davis,

United States

*Correspondence:

Rong Gao

714866001@qq.com

Baoqi Dang

Doctor_Dang82@hotmail.com

Specialty section:

This article was submitted to

Stroke,

a section of the journa

Frontiers in Neurology

Received: 10 August 2020 Accepted: 05 November 2020 Published: 07 December 2020

Citation:

Wu M, Gao R, Dang B and Chen G (2020) The Blood Component Iron

Causes Neuronal Apoptosis Following

Intracerebral Hemorrhage via the

PERK Pathway.

Front. Neurol. 11:588548.

doi: 10.3389/fneur.2020.588548

\section{The Blood Component Iron Causes Neuronal Apoptosis Following Intracerebral Hemorrhage via the PERK Pathway}

\author{
Muyao $\mathrm{Wu}^{1}$, Rong $\mathrm{Gao}^{2 *}$, Baoqi Dang ${ }^{1 *}$ and Gang Chen ${ }^{3}$ \\ ' Department of Rehabilitation, Zhangjiagang TCM Hospital Affiliated to Nanjing University of Chinese Medicine, Suzhou, \\ China, ${ }^{2}$ Department of Neurosurgery, Zhangjiagang TCM Hospital Affiliated to Nanjing University of Chinese Medicine, \\ Suzhou, China, ${ }^{3}$ Department of Neurosurgery and Brain and Nerve Research Laboratory, The First Affiliated Hospital of \\ Soochow University, Suzhou, China
}

PERK signaling pathway plays an important role in neuronal apoptosis after Intracerebral hemorrhage $(\mathrm{ICH}) . \mathrm{ICH}$ can cause the release of blood components into the brain. However, which component in the blood plays a major role still unclear. This study was designed to investigate the activation of the PERK pathway in different blood components after $\mathrm{ICH}$ and explore which components have major relationships with neuronal apoptosis. Eighty-five Sprague-Dawley rats were used to establish an $\mathrm{ICH}$ model. Western blot (WB) and immunofluorescence (IF) were used to evaluate the expression of the PERK pathway. TUNEL staining, FJC staining and neurological score were used to evaluate neuronal apoptosis and necrosis after $\mathrm{ICH}$. The results showed that protein levels of $\mathrm{p}$-PERK and $\mathrm{p}$-elF2 $\alpha$ were upregulated following $\mathrm{ICH}$ with the injection of $\mathrm{Fe}^{3+}$ and $\mathrm{Fe}^{2+}$ after $48 \mathrm{~h}$. Then, deferoxamine (DFX) was used to study the roles of $\mathrm{Fe}^{3+}$ in $\mathrm{ICH}$ through the PERK signaling pathway. The results showed that injection of DFX reversed increasing protein levels and prevented neuronal apoptosis. Thus, iron plays an important role in $\mathrm{ICH}$ through the PERK signaling pathway. Furthermore, the reduction of iron demonstrates neuroprotective effects in $\mathrm{ICH}$. This suggests that targeting intervention of the iron and PERK pathway could be an effective treatment strategy to improve $\mathrm{ICH}$ prognosis.

Keywords: blood components, iron, intracerebral hemorrhage, PERK pathway, apoptosis

\section{INTRODUCTION}

Intracerebral hemorrhage (ICH), a common subtype of hemorrhagic stroke, has high morbidity/mortality with an estimated yearly worldwide prevalence of $16 / 100,000$ (1). Studies have shown that inflammation, oxidative stress, cytotoxicity and excitotoxicity caused by plasma components and red blood cell (RBC) lysates were the main factors causing secondary brain injury (SBI) following $\mathrm{ICH}$, ultimately leading to brain edema and brain parenchymal damage $(2,3)$. ICH can cause disorders in cell metabolism, activating endoplasmic reticulum stress (ERs) and the unfolded protein response (UPR), eventually reestablish cellular homeostasis. The cell death program is activated when too much stress to restore homeostasis (4). 
ICH can cause the release of blood components into the brain, such as erythrocytes and fibrinogen. Erythrocytes start to lyse 1 day following ICH and continues over days to weeks (5). When erythrocytes break down, hemoglobin $(\mathrm{Hb})$ is metabolized into heme, which is further degraded into biliverdin, ferrous iron, and $\mathrm{CO}$ by heme oxygenase- $1 \mathrm{HO}-1)$ and heme oxygenase2 (HO-2) $(6,7)$. Lysis of RBCs leads to an increase in iron content surrounding the hematoma. Iron can initiate the release of large amounts of reactive oxygen species (ROS), induce the production of lipid ROS. The excessive accumulation of lipid ROS causes intracellular oxidative stress response, which leads to the damage of proteins and lipids, and ultimately results in neuronal ferroptosis and cell death. Additionally, it can lead to secondary brain injuries such as brain edema, intracranial hematoma and irreversible neurological deficits following $\mathrm{ICH}$ (8-10). It has been indicated that RBC lysis, with the release of iron, play a significant role in the development of hydrocephalus after intraventricular hemorrhage (11). The cytotoxicity of iron generated by hemolysis is associated with oxidative stress and inflammatory response (12). Studies over the past decade had found that prevention of iron-mediated cytotoxicity is significant for the treatment of SBI after ICH. Chelation of iron with deferoxamine (DFX) reduced brain injury in animals $(13,14)$.

ERs caused by misfolded/unfolded accumulation of proteins is one of the main mechanisms leading to neuronal apoptosis. When ERs occurs, UPR can remove misfolded/unfolded proteins, allowing it to possibly play a significant role in cell survival $(15,16)$. The UPR is triggered by three types of ER stress sensor proteins: PERK, inositol requiring kinase 1 (IRE1) and activating transcription factor six (ATF6). PERK acts as a central pressure sensor in ER, restoring homeostasis by performing adaptive programs through protein translation and induction of the activating ATF4 (17). The inhibition of PERK signaling pathway can reduce SBI following ICH by suppressing apoptosis $(18,19)$. However, it is not clear which component of the hematoma plays the key role following ICH and whether it is related to the PERK pathway activated by the UPR.

Given its widespread prevalence and high mortality/morbidity, exploration of the pathogenesis and mechanisms of ICH, as well as identification of new biomarkers and therapeutic targets is of the utmost importance. This study investigated the roles of different blood components, especially iron, in SBI following ICH, via the PERK signaling pathway. Specifically, a combination of DFX and $\mathrm{Fe}^{3+}$ was used to investigate whether iron in the blood plays an important role in neuronal apoptosis following ICH.

\section{MATERIALS AND METHODS}

\section{Experimental Design}

In Experiment 1, animal weights, food intake, and motor abilities were similar in all rats. Twenty eight rats (28 out of 33) were randomized to seven groups using computer-based randomization, including Sham, $\mathrm{ICH}, \mathrm{ICH}+\mathrm{Fe}^{3+}, \mathrm{ICH}+\mathrm{Fe}^{2+}$, $\mathrm{ICH}+$ Hemoglobin, $\mathrm{ICH}+$ Bilirubin and $\mathrm{ICH}+\mathrm{NaCl}$. $\mathrm{NaCl}$ was used as vehicle. At $48 \mathrm{~h}$ after ICH (18), the rats were sacrificed and cerebral tissues were collected around the damaged areas. Brain tissues surrounding the damaged (Figure 1A) were sampled to perform Western blot (WB) and Immunofluorescence (IF) analysis to assess the expression of PERK, p-PERK, eIF2 $\alpha$, peIF2 $\alpha$ (Figure 1B).

In Experiment 2, in order to assess the function of DFX in ICH caused by iron, 48 rats ( 48 out of 52) were randomized to four groups using computer-based randomization, including Sham, $\mathrm{ICH}+\mathrm{Fe}^{3+}, \mathrm{ICH}+\mathrm{Fe}^{3+}+$ Vehicle, and $\mathrm{ICH}+\mathrm{Fe}^{3+}+$ DFX. At $48 \mathrm{~h}$ after $\mathrm{ICH}$, the rats were sacrificed after neurological scoring and cerebral tissues were collected around the damaged areas. Brain tissues were used for $\mathrm{WB}$ to measure the expression of PERK, p-PERK, eIF2 $\alpha$, p-eIF2 $\alpha$, ATF4 and caspase-3. Fluorojade C(FJC) and TUNEL staining were performed for detecting necrosis and neuronal apoptosis. Wet-dry method was used to measure the brain edema (Figure 1C).

\section{Animals}

Here, totally 85 male Sprague-Dawley rats (250-300g) were purchased from JOINN Laboratories, Suzhou, China. Of these, 76 were analyzed. Animals were housed in a controlled temperature and humidity room with a $12 \mathrm{~h}$ light/dark cycles and free access to food and water. The animal studies had approval from the Institutional Animal Care and Use Committee of Nanjing University of Chinese Medicine, and followed all relevant national and international guidelines.

\section{ICH Model}

The ICH model in rats was established by autologous whole blood stereotaxic injection or several kinds of blood components according to previous reports with some modifications (11, 20). In brief, rats were anesthetized with intraperitoneally administered pentobarbitone $(40 \mathrm{mg} / \mathrm{kg})$ and mounted on a stereotaxic apparatus (SA-100, Shanghai Yuyan Instruments, China). A cranial burr hole $(0.2 \mathrm{~mm}$ anterior to bregma, $3.5 \mathrm{~mm}$ lateral to midline and $5.5 \mathrm{~mm}$ ventral to cortex) was drilled corresponding to the right basal ganglia. After collected by cardiac puncture, autologous whole blood $(100 \mu \mathrm{L})$ was injected slowly $(20 \mu \mathrm{L} / \mathrm{min})$ into basal ganglia with a microinjector (Hamilton, USA). At the same time, $\mathrm{FeCl}_{3}(1 \mathrm{mmol} / \mathrm{L}, 100 \mu \mathrm{L})$, $\mathrm{FeCl}_{2}(1 \mathrm{mmol} / \mathrm{L}, 100 \mu \mathrm{L})$, Hemoglobin $(300 \mathrm{mg} / \mathrm{mL}, 100 \mu \mathrm{L})$, Bilirubin $(12 \mathrm{mg} / \mathrm{mL}, 100 \mu \mathrm{L})$ and $\mathrm{NaCl}(100 \mu \mathrm{L})$ were also injected under the same conditions (11). Kept the needle in place for $5 \mathrm{~min}$. After sealing the burr hole with bone wax, the scalp was disinfected and sutured. Rats in Sham group were only subjected to a cranial burr hole without injection. The rats were sacrificed $48 \mathrm{~h}$ after ICH according to previous study (18).

\section{Drug Injection}

DFX (iron chelator, $100 \mathrm{mg} / \mathrm{kg}$, MCE, USA) was intraperitoneally injected 2 and $6 \mathrm{~h}$ after ICH, followed by every $12 \mathrm{~h}$ until the rats were sacrificed for the material. DFX can bind free iron in a stable compound, preventing it from taking part in chemical reactions. The $\mathrm{Fe}^{3+}+$ vehicle group was given $\mathrm{NaCl}$ for the same time course and dosage. 


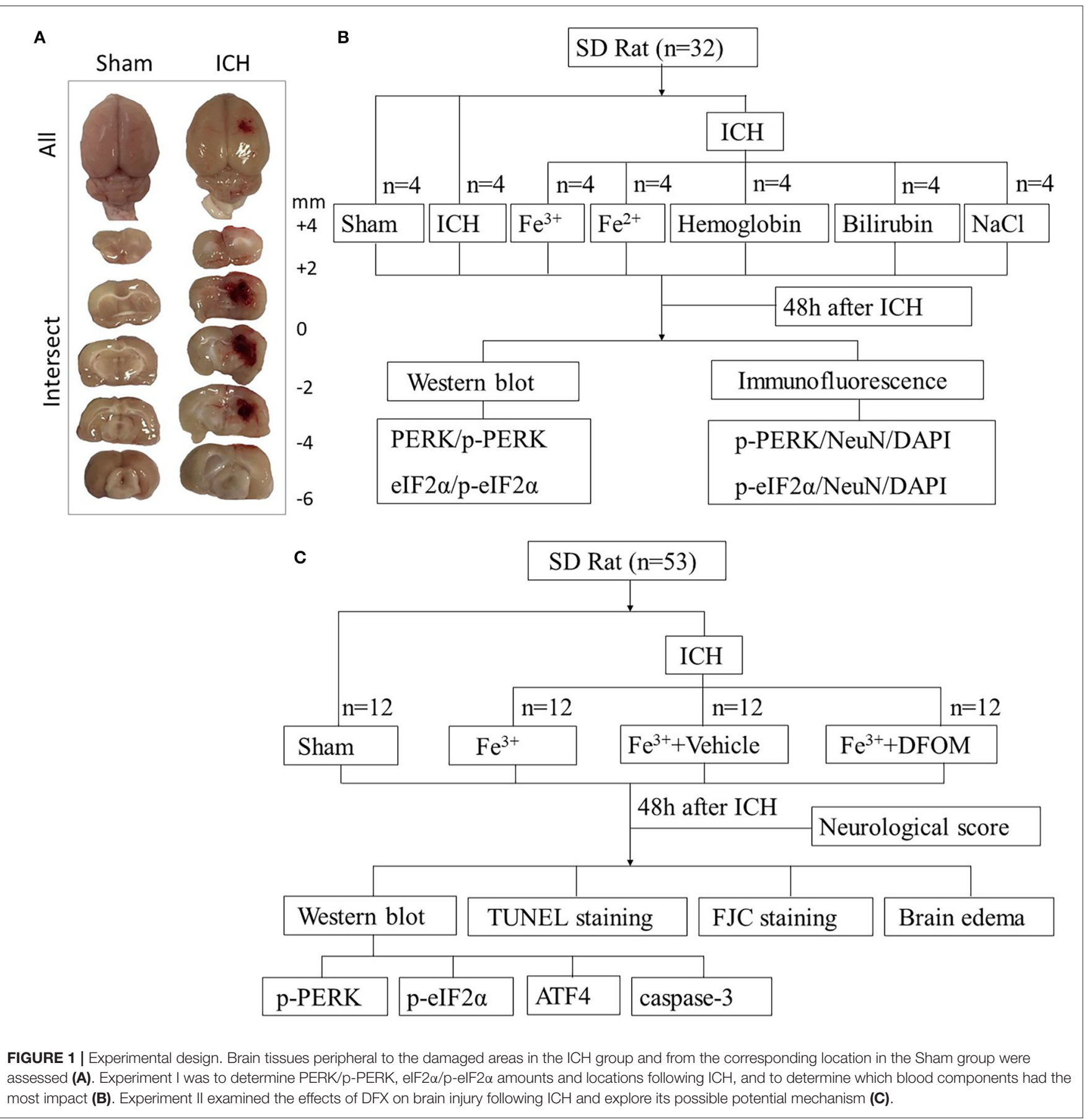

\section{Western Blot}

WB was performed as described (21). Briefly, cortex tissues were lysed with RIPA Lysis Buffer containing Protease inhibitor cocktail (Beyotime, China), and centrifuged at $12,000 \mathrm{~g}$ at $4^{\circ} \mathrm{C}$ for $15 \mathrm{~min}$. The supernatant was collected and determined with Pierce ${ }^{\mathrm{TM}}$ BCA Protein Assay Kit (Thermo Fisher, USA). After mixed with $5 \mathrm{X}$ loading buffer (Beyotime, China), the supernatant was bathed at $100^{\circ} \mathrm{C}$ for $5 \mathrm{~min}$. Equal amounts of extracted proteins were separated by SDS-polyacrylamide gels (Beyotime, China) and transferred onto PVDF membranes (Millipore, USA). After blocking with QuickBlock ${ }^{\mathrm{TM}}$ Blocking Buffer (Beyotime, China) at room temperature (RT) for $30 \mathrm{~min}$, membranes were incubated at $4{ }^{\circ} \mathrm{C}$ overnight with the following rabbit primary antibodies: anti-PERK (Abcam, USA), anti-pPERK (Cell Signaling, USA), rabbit anti-eIF2 $\alpha$ (Abcam), antip-eIF2 $\alpha$ (1:500, Abcam), anti-ATF4 (1:1,000, Abcam) and anticaspase-3 (1:500, Abcam). Mouse anti- $\beta$-actin (1:10,000, Sigma, USA) was used as control. Then the membranes were incubated 
with goat anti-rabbit IgG-HRP (Invitrogen, USA) and goat anti-mouse IgG-HRP (Invitrogen) for $1 \mathrm{~h}$ at RT. Detection used Immobilon $^{\mathrm{TM}}$ Western Chemiluminescent HRP Substrate (Millipore) and a LUMINESCENT IMAGE ANALYZER (GE Healthcare Bio-Sciences, Sweden). ImageJ (National Institutes of Health, USA) was used to analyze data.

\section{Immunofluorescence}

Double IF staining was performed as described (22). Briefly, brain tissues were fixed with $4 \%$ paraformaldehyde, embedded in paraffin, and cut into $5 \mu \mathrm{m}$. After dewaxing, the sections were permeabilized in Immunostaining Permeable Buffer (Beyotime). After three washes in PBS, the sections were blocked in Immunostaining Blocking Buffer (Beyotime) for $1 \mathrm{~h}$ at $\mathrm{RT}$ and incubated with primary antibodies at $4{ }^{\circ} \mathrm{C}$ overnight: rabbit anti-p-PERK (Abcam), rabbit anti-p-eIF2 $\alpha$ (Abcam), mouse anti-NeuN (Abcam). After three washes in PBS, the sections were incubated with donkey anti-rabbit IgG Alexa Fluor 488 (Invitrogen) and donkey anti-mouse IgG Alexa Fluor 555 (Invitrogen), for $1 \mathrm{~h}$ at RT. Finally, counterstaining was carried out with DAPI and a U-RFL-T fluorescence microscope (OLYMPUS, Japan) was utilized for analysis.

\section{TUNEL Staining}

TUNEL staining was carried out to detect apoptosis in intracerebral cells (18). The sections were dewaxed and incubated in proteinase $\mathrm{K}$ at $37^{\circ} \mathrm{C}$ for $20 \mathrm{~min}$. After three washes in PBS, the sections were incubated in TUNEL detection liquid (Beyotime) at $37^{\circ} \mathrm{C}$ for $1 \mathrm{~h}$. After counterstained with DAPI, the TUNELpositive neurons were observed under a U-RFL-T fluorescence microscope (OLYMPUS).

\section{Fluoro-Jade C Staining}

FJC staining was performed according to the manufacturer's instructions (Biosensis, South Australia). Briefly, the sections were dewaxed and incubated with potassium permanganate (1:10 in distilled water) for $10 \mathrm{~min}$. Rinse the sections for $2 \mathrm{~min}$ in distilled water. Then the sections were incubated with FJC (1:10 in distilled water) in the dark for $10 \mathrm{~min}$. After 3 rinses for $1 \mathrm{~min}$ in distilled water, the sections were dried at $60^{\circ} \mathrm{C}$ for $5 \mathrm{~min}$, soaked in xylene for $1 \mathrm{~min}$, and coverslipped with Neutral Balsam Mounting Medium (Yeasen Biotech. China). The FJC-positive cells were photographed by a U-RFL-T fluorescence microscope (OLYMPUS) and the total number of FJC-positive cells was the average of three high-power fields.

\section{Brain Edema}

The wet-dry method was carried out for assessing brain edema in the injured brain $(23,24)$. Briefly, the brains were separated into symmetrical sides and their wet weights were immediately obtained. Then the samples underwent drying at $100^{\circ} \mathrm{C}$ for $72 \mathrm{~h}$ (or until the dried weights no longer change), followed by dry weight measurements. Brain water content (\%) was derived as [(wet weight-dry weight)/(wet weight)] $\times 100 \%$.

\section{Neurological Scoring}

Neurological deficiency was conducted at $48 \mathrm{~h}$ post-ICH based on the modified Garcia score consisted of seven components (25,
26). Scores in each subtest ranged between 0 and 3 (maximum total score of 21, indicating no neurological defects).

\section{Statistical Analysis}

All data are mean \pm SD. Data analysis was carried out with GraphPad Prism 8.0 software (San Diego, USA). One-way ANOVA was used for multiple comparisons, and Student-Newman-Keuls post-hoc test was used to determine the differences between groups. $p<0.05$ indicated statistical significance.

\section{RESULTS}

\section{PERK/p-PERK, elF2 $\alpha / p-e l F 2 ~ \alpha$ Protein Levels in Brain Tissues Following ICH Caused by Several Blood Components}

WB showed that, compared with the Sham group, p-PERK and p-eIF $2 \alpha$ protein amounts increased in the $\mathrm{ICH}, \mathrm{ICH}+\mathrm{Fe}^{3+}$, and $\mathrm{ICH}+\mathrm{Fe}^{2+}$ groups. However, other groups demonstrated little changes (Figure 2). Consistent with WB analysis, IF staining revealed that there were numerous p-PERK-positive neurons and p-eIF $2 \alpha$-positive neurons in ICH, ICH $+\mathrm{Fe}^{3+}$, and $\mathrm{ICH}$ $+\mathrm{Fe}^{2+}$ groups at $48 \mathrm{~h}$ following ICH compared with the Sham group (Figure 3).

\section{DFX Intervention Through the Perk Pathway Following ICH}

After DFX intervention, the protein level of p-PERK and p-eIF2 $\alpha$ was significantly decreased in the $\mathrm{Fe}^{3+}+\mathrm{DFX}$ group compared with the $\mathrm{ICH}+\mathrm{Fe}^{3+}$ group. The protein level of $\mathrm{Fe}^{3+}+$ Vehicle group was similar to the $\mathrm{ICH}+\mathrm{Fe}^{3+}$ group. Additionally, ATF4 protein levels were higher in the $\mathrm{Fe}^{3+}$ and $\mathrm{Fe}^{3+}+$ Vehicle groups than in the Sham group. After DFX intervention, ATF4 level was significantly lower in the $\mathrm{Fe}^{3+}+\mathrm{DFX}$ group compared with the $\mathrm{ICH}+\mathrm{Fe}^{3+}$ group (Figure 4).

\section{Neuronal Degeneration and Neuronal Death Following ICH in Rats Administered DFX}

Protein levels of Caspase-3 were lower following DFX intervention when compared to the $\mathrm{ICH}+\mathrm{Fe}^{3+}$ group (Figures 4A,E). Additionally, the degree of neuronal apoptosis (TUNEL) in the $\mathrm{ICH}+\mathrm{Fe}^{3+}$ group was significantly greater in comparison with that of Sham group. There was no difference in neuronal apoptosis between the $\mathrm{ICH}+\mathrm{Fe}^{3+}$ and $\mathrm{Fe}^{3+}+$ Vehicle groups, while it was significantly lower in the $\mathrm{Fe}^{3+}+\mathrm{DFX}$ group when compared to the $\mathrm{ICH}+\mathrm{Fe}^{3+}$ group (Figure 5A). The degree of neuronal necrosis (FJC) was consistent with the degree of neuronal apoptosis (Figure 5B).

\section{Neurological Behavioral Scores and Brain Edema in ICH Rats Following DFX Intervention}

Neurological behavioral scores from a modified Garcia test were starkly decreased in the $\mathrm{ICH}+\mathrm{Fe}^{3+}$ group when compared to the Sham group, while the $\mathrm{ICH}+\mathrm{Fe}^{3+}$ group and $\mathrm{Fe}^{3+}+$ Vehicle 


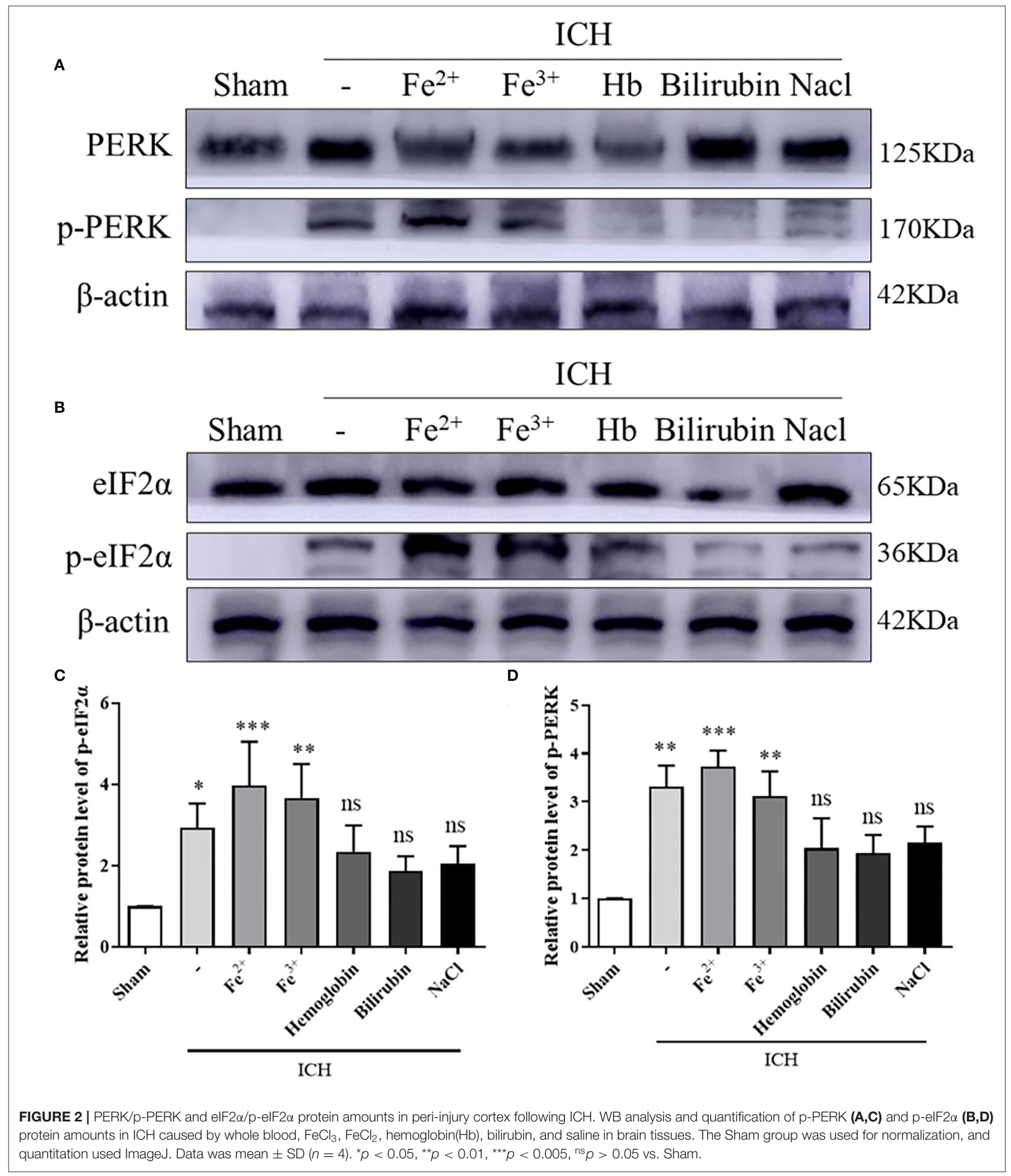

group had comparable scores. The $\mathrm{Fe}^{3+}+\mathrm{DFX}$ group had overtly ameliorated neurological behavioral scores in comparison with the $\mathrm{ICH}+\mathrm{Fe}^{3+}$ group (Figure 5F). Additionally, brain edema was markedly reduced in the injured hemispheres following DFX intervention post-ICH. However, brain edema had no significant changes in the contralateral brain (Figure 5E). 

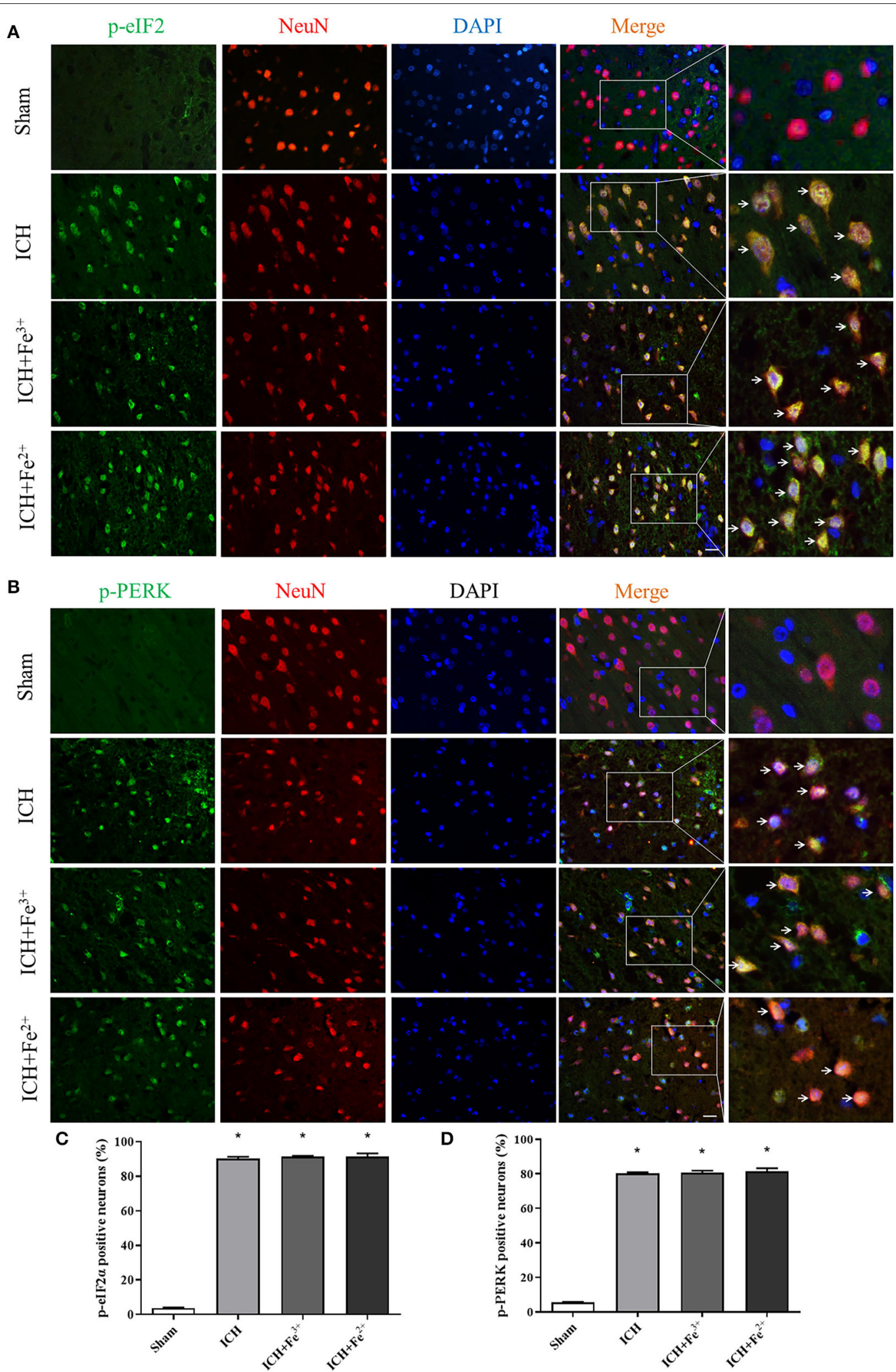

FIGURE 3 | Post-ICH IF in rat brain tissues. Double immunofluorescence micrographs showing green-labeled p-elF2 $\alpha$ (A,C)/p-PERK (B,D) and red-labeled NeuN neurons surrounding the damaged brain tissue. Counterstaining utilized DAPI (blue). Scale bar $=50 \mu \mathrm{m}$. Data was mean $\pm \mathrm{SD}(n=4)$. ${ }^{*} p<0.05$ vs. Sham. 
A
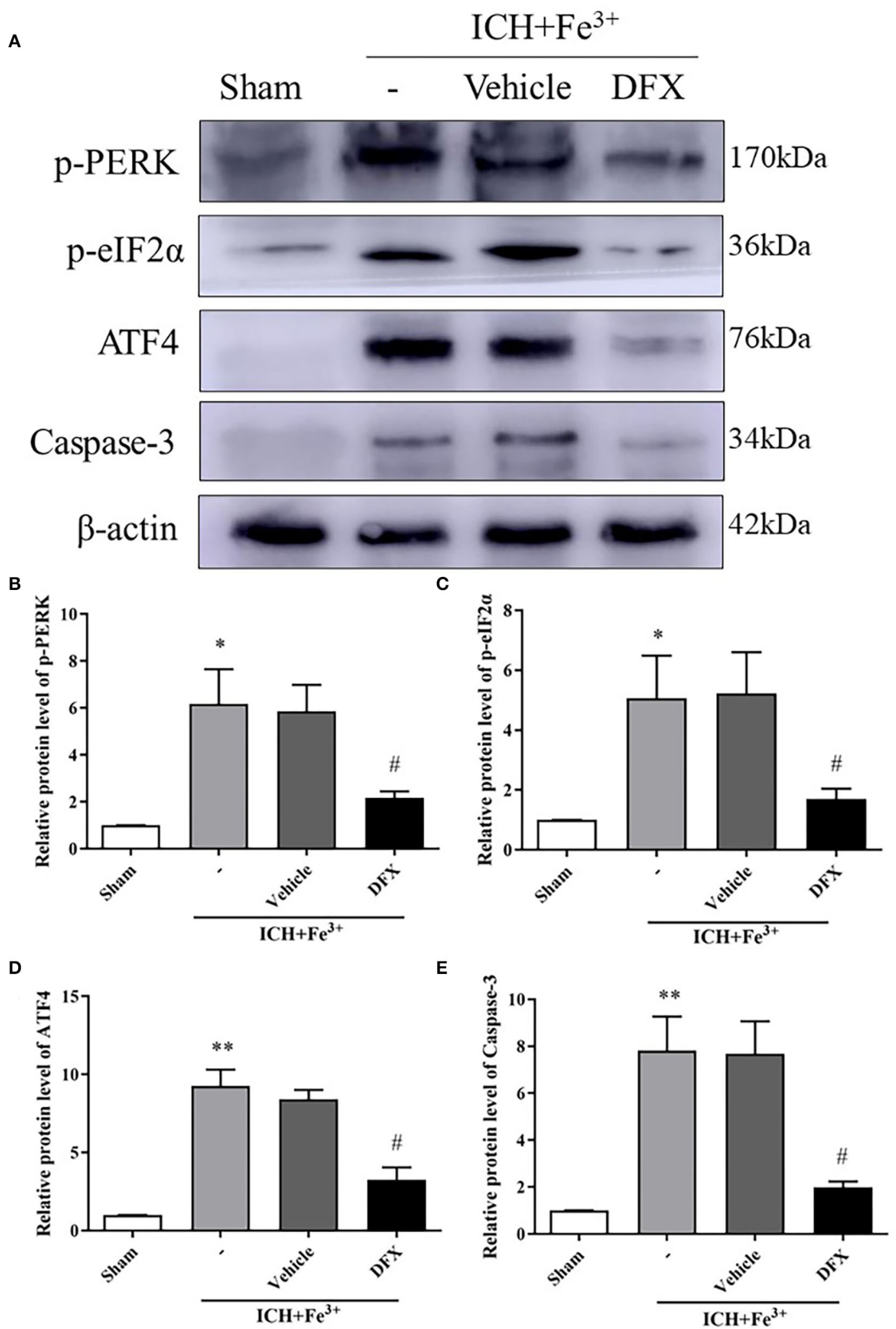

FIGURE 4 | Effect of the intervention of DFX following ICH. WB analysis and quantification of p-PERK (A,B), p-elF2 $\alpha$ (A,C), ATF4 (A,D) and Caspase-3 (A,E) protein levels in $\mathrm{ICH}$ caused by $\mathrm{Fe}^{3+}$ in brain tissues. The Sham group was used for normalization, and quantitation used ImageJ. Data was mean $\pm \mathrm{SD}(n=4)$. ${ }^{*} p<0.05$, ${ }^{* *} p<0.01$ vs. Sham, $\# p<0.05$ vs. $\mathrm{ICH}+\mathrm{Fe}^{3+}$ group. 
A

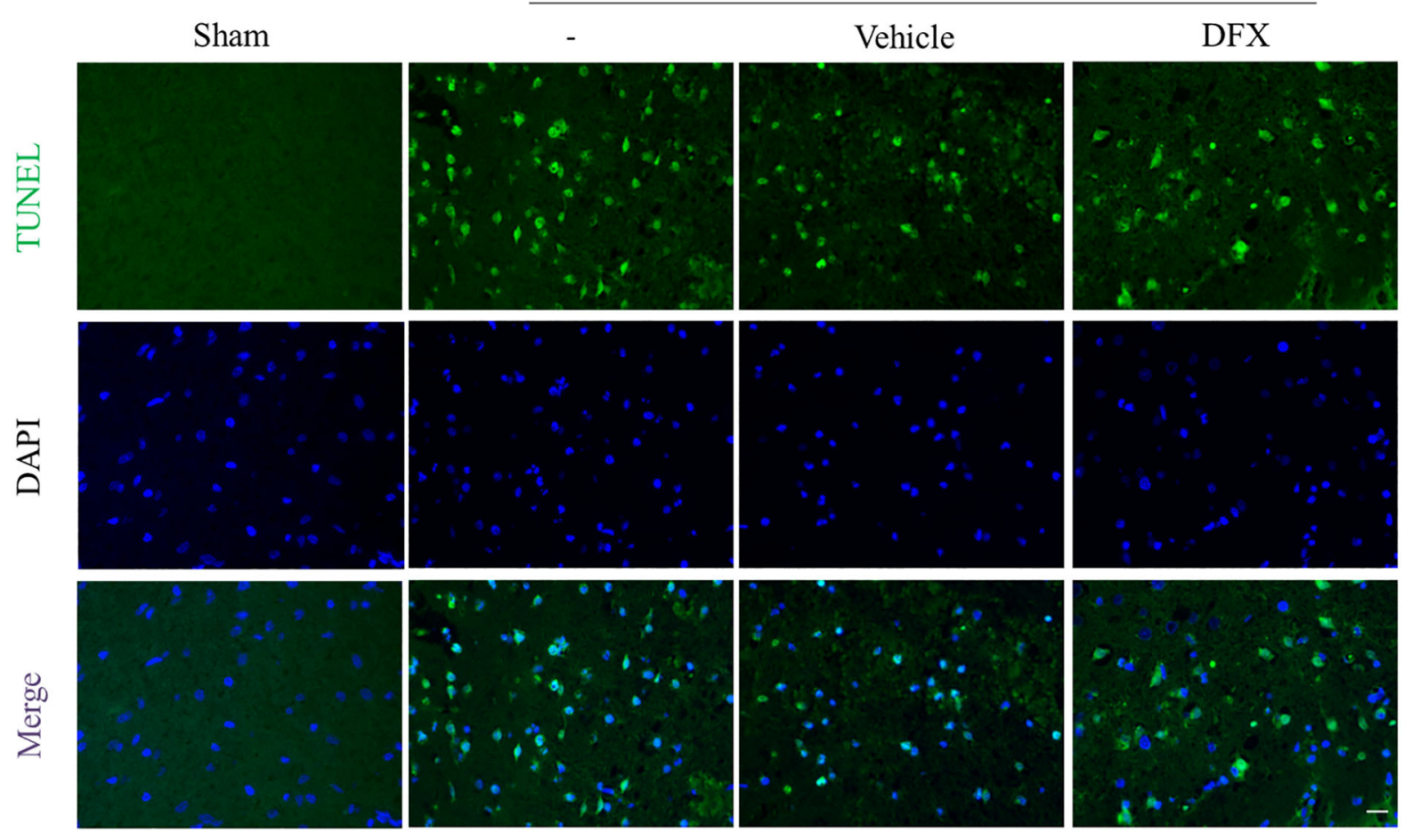

B

$\mathrm{ICH}+\mathrm{Fe}^{3+}$

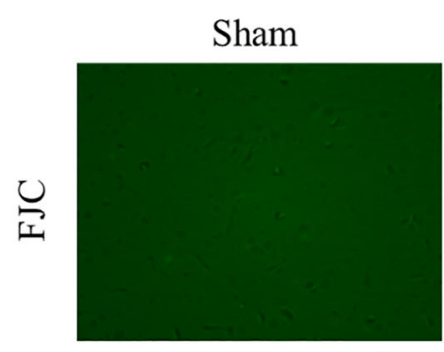

C

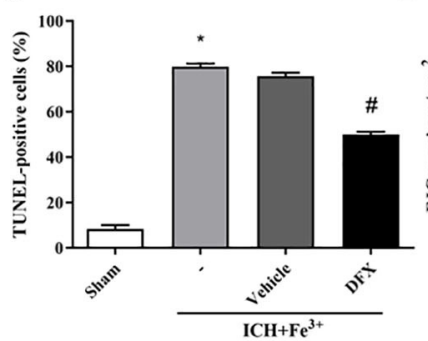

D

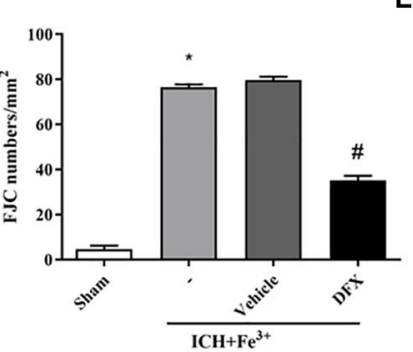

\section{Vehicle}

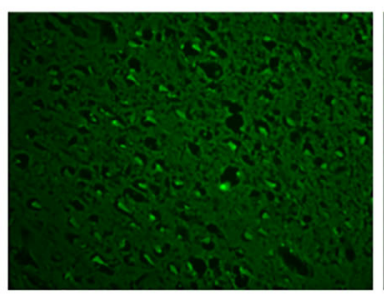

E

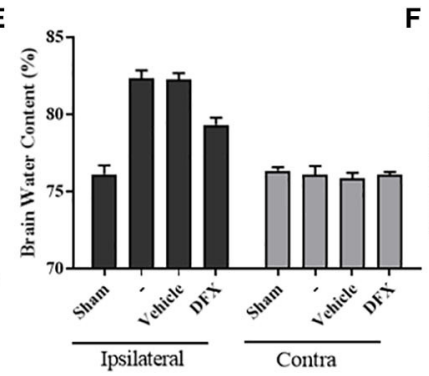

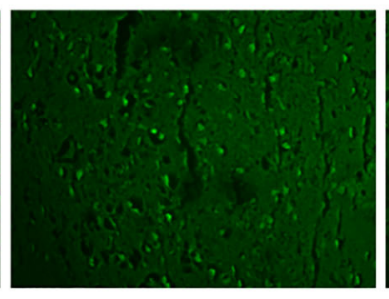

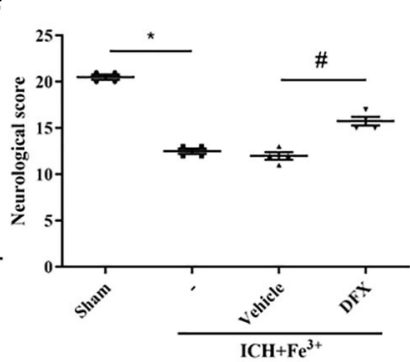

FIGURE 5 | Effect of the intervention of DFX on PERK signaling pathway $48 \mathrm{~h}$ following ICH. TUNEL-positive neurons (A,C) in each group following DFX administration. Scale bar $=50 \mu \mathrm{m}$. FJC-positive cells (B,D) at $48 \mathrm{~h}$ in each group. Scale bar $=100 \mu \mathrm{m}$. Brain water amounts of the bilateral hemispheres were assessed by the wet-dry technique (E). Neurological behavioral scores in each group (F). Data was mean $\pm \operatorname{SD}(n=4)$. ${ }^{\star} p<0.05$ vs. Sham, $\# p<0.05$ vs. ICH+Fe ${ }^{3+}$ group.

\section{DISCUSSION}

In this study, we found that iron plays a significant role in the occurrence of ICH-induced SBI. After ICH caused by autologous whole blood or other blood components (especially $\mathrm{Fe}^{3+}$ and $\mathrm{Fe}^{2+}$ ), ERs activated the PERK pathway and increased $\mathrm{p}$-PERK, p-eIF $2 \alpha$ and ATF4 protein levels. The PERK pathway blocked the initiation process of translation and synthesis of protein, leading 


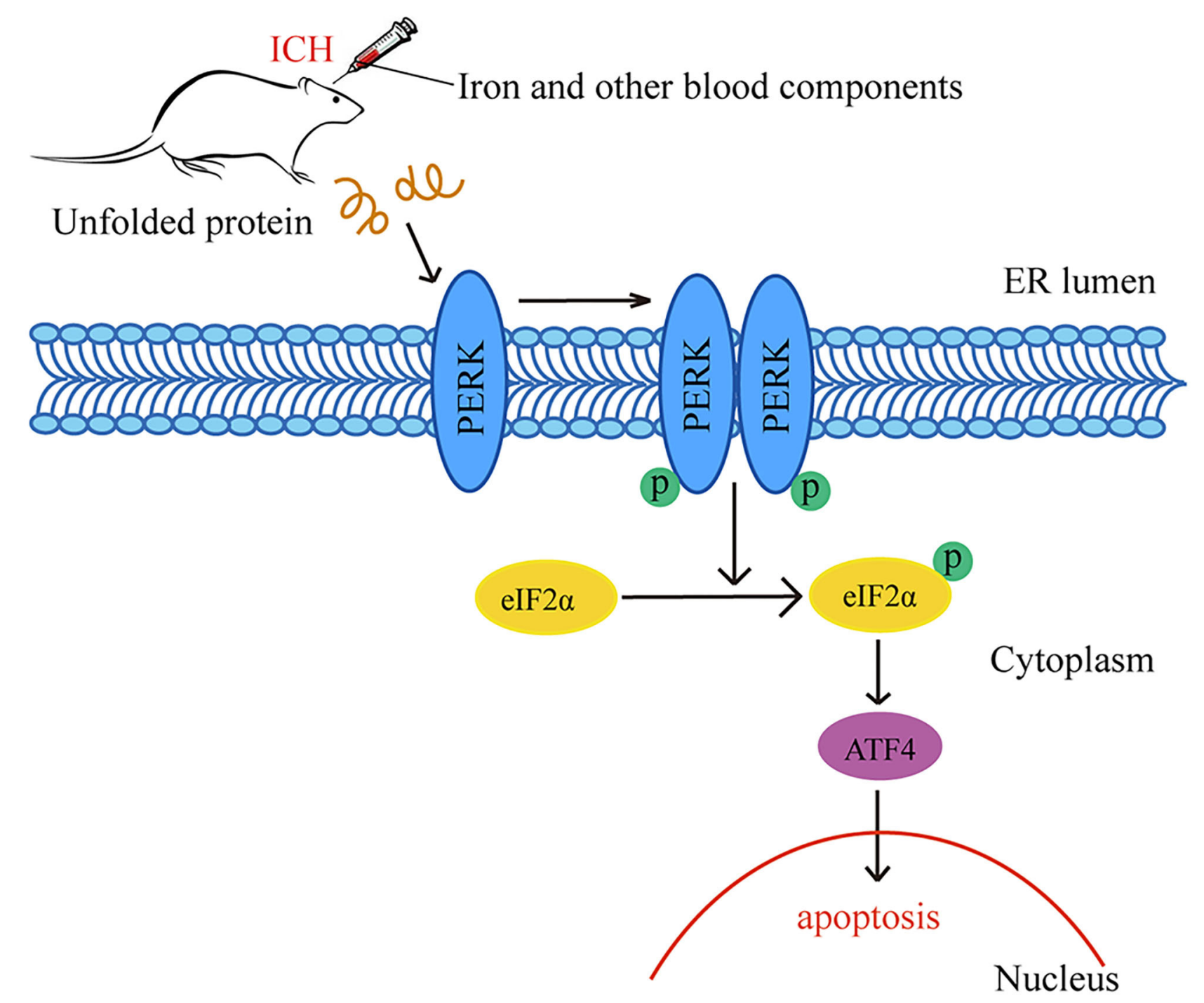

FIGURE 6 | Schematic representation of potential mechanisms of the neuroprotective effects of PERK pathway after ICH. Following ICH caused by autologous whole blood or other blood components (especially $\mathrm{Fe}^{3+}$ and $\mathrm{Fe}^{2+}$ ), the activation of ER stress activates the PERK pathway and increases the protein amounts of $\mathrm{p}-\mathrm{PERK}$, p-elF2 $\alpha$ and ATF4. The activation of PERK pathway blocks the initiation process of translation and synthesis of protein, leading to neuronal apoptosis.

to neuronal apoptosis (Figure 6). We used DFX to interfere with $\mathrm{Fe}^{3+}$. It showed that DFX could prevent neuronal apoptosis by inhibiting the PERK pathway and alleviate ERs.

Studies have reported that, ERs-induced neuronal death is one of the important causes of brain injury. Cells activate a series of adaptive processes through UPR to restore the function of ER when ERs occurs. As a part of the UPR, the PERK pathway may be associated with a series of pathophysiological processes in neurological diseases (27). As we have previously reported, in early ICH-induced SBI, PERK activation is related to oligomerization and trans-autophosphorylation when unfolded or misfolded proteins accumulate, and p-PERK specifically induces eIF $2 \alpha$ to p-eIF $2 \alpha$ at ser51 to upregulate ATF4 (18, 19). However, it is unclear which components of blood are involved in the development of ICH through the PERK pathway. Previous studies did not address the effects of various blood components on post-ICH neuronal apoptosis via the PERK pathway. Therefore, in this study, we explored these effects. Specifically, we investigated the pathophysiological role of multiple components produced after hematoma decomposition in ICH and the relationship between blood components and the PERK pathway.

As we all know, blood components include hemolyzed erythrocytes, methemoglobin, ferritin, chloride, fibrinogen, thrombin, and hemin (28). The effects of hemoglobin, iron, and other blood components on brain tissue in damaged areas are considered to be the influencing factors of SBI. Brain edema was observed after the injection of lysed blood cells into the 
ICH model, while not observed following injection of packed erythrocytes, suggesting the lysed products of RBC were the main cause of brain injury (29). In our study, we used hemoglobin, iron $\left(\mathrm{Fe}^{3+}\right.$ and $\left.\mathrm{Fe}^{2+}\right)$ and bilirubin to conduct an $\mathrm{ICH}$ rat model. Protein expressions of p-PERK and p-eIF2 $\alpha$ were increased in the iron-treated rats. Which was consistent with it in the rats treated with autologous whole blood (Figure 2). This suggests that iron plays a role after $\mathrm{ICH}$, and this is consistent with previous reports.

Here, we found that both $\mathrm{Fe}^{2+}$ and $\mathrm{Fe}^{3+}$ can cause brain injury. The p-PERK, p-eIF2 $\alpha$ and ATF4 expression can be reduced by the use of DFX chelating iron to silence the PERK pathway, and reduce the brain injury (Figure 4). It has been reported that, blood components such as iron and thrombin play a significant role in brain injury following ICH (30). RBC lysate contains hemoglobin, as well as its components, iron and heme. The form of the iron within heme can be either $\mathrm{Fe}^{2+}$ or $\mathrm{Fe}^{3+}$, both of which can cause brain injury (31). Under normal conditions, iron is present in the form of $\mathrm{Fe}^{3+}$ under dynamic regulation by iron transporters on cell membranes, or stored on proteins to form transferrin and ferritin. However, in the case of pathological damage to the regulatory system of the cell, the increased $\mathrm{Fe}^{3+}$ is readily converted to $\mathrm{Fe}^{2+}$ by the electron transfer response (32). The degradation of hemoglobin causes the release of iron when RBCs are lysed. The concentration of iron surrounding the perihematomal brain tissue can reach as high as $10 \mathrm{mmol} / \mathrm{L}$, causing obvious brain damage (33). Based on previous studies, we demonstrated that iron can cause more severe nerve damage and brain edema compared to a variety of other blood components (Figure 5). Therefore, we selected iron as the intervention target, and combined DFX with $\mathrm{Fe}^{3+}$ to explore the effect of these on brain injury and brain edema through the PERK pathway. This points out the importance of $\mathrm{Fe}^{3+}$ in hydrocephalus and brain damage after erythrolysis.

Neuronal necrosis, apoptosis, and destruction of the BBB are the main components of brain injury following $\mathrm{ICH}$. The destruction of the $\mathrm{BBB}$ can lead to the formation of brain edema, neuronal death, and neurological impairment. Iron from blood degradation is associated with BBB dysfunction after hemorrhagic stroke (34). It has shown that intraventricular injection of iron can cause hydrocephalus. The chelation of iron and DFX is able to reduce hydrocephalus caused by RBC lysis (11). In this study, following ICH with the injection of iron, BBB permeability was increased, and brain edema was aggravated, leading to neuronal necrosis and apoptosis. Using DFX following $\mathrm{ICH}$ can reverse these results, resulting in reduced cerebral edema, nerve necrosis, and apoptosis (Figure 5).

\section{REFERENCES}

1. Wilkinson DA, Pandey AS, Thompson BG, Keep RF, Hua Y, $\mathrm{Xi}$ G. Injury mechanisms in acute intracerebral hemorrhage. Neuropharmacology. (2018) 134:240-8. doi: 10.1016/j.neuropharm.2017.0 9.033

2. Egashira Y, Hua Y, Keep RF, Xi G. Intercellular cross-talk in intracerebral hemorrhage. Brain Res. (2015) 1623:97109. doi: 10.1016/j.brainres.2015.04.003
The current study has several limitations. First, in this study, we only focused on adult male rats, despite ICH being common in older females (35). Second, we focused on the PERK pathway, ignoring the other two UPR pathways (IRE1 pathways and ATF6 pathway). Third, iron comes in two forms, $\mathrm{Fe}^{3+}$ and $\mathrm{Fe}^{2+}$, and both are important during ICH. However, we only used DFX to chelate $\mathrm{Fe}^{3+}$, as $\mathrm{Fe}^{2+}$ does not have a specific inhibitor. In future research, we can attempt to find more specific methods to intervene $\mathrm{Fe}^{2+}$ to further explore its effect on ICH. Additionally, the specific initiation mechanism of the UPR pathway following $\mathrm{ICH}$ also needs further study.

In conclusion, this study provides evidence that various blood components, especially iron, the lysed product of RBCs, can lead to neuronal apoptosis and induce brain edema. This may be an effective therapeutic target for ICH. Furthermore, the effects of iron in SBI following ICH may be related to the PERK pathway.

\section{DATA AVAILABILITY STATEMENT}

The original contributions generated for this study are included in the article/supplementary material, further inquiries can be directed to the corresponding author/s.

\section{ETHICS STATEMENT}

The animal study was reviewed and approved by Institute of Animal Care Committee of Zhangjiagang Traditional Chinese Medicine Hospital.

\section{AUTHOR CONTRIBUTIONS}

BD and GC: study conception and design. MW and RG: data collection and analysis. MW: writing of the manuscript. All authors have assessed the totality of study data, and are responsible for their integrity and accurate analysis.

\section{FUNDING}

This study was supported by Suzhou Gusu Health personnel Training Project (GSWS2019076) and Zhangjiagang Science and Technology Support Plan project (ZKS1712).

\section{ACKNOWLEDGMENTS}

We are grateful to LetPub (www.letpub.com) for help in language editing of the present manuscript. oxidative stress, and antioxidant therapy. Oxid Med Cell Longev. (2016) 2016:1203285. doi: 10.1155/2016/1203285

4. Niu M, Dai X, Zou W, Yu X, Teng W, Chen Q, et al. Autophagy, endoplasmic reticulum stress and the unfolded protein response in intracerebral hemorrhage. Transl Neurosci. (2017) 8:37-48. doi: 10.1515/tnsci-2017-0008

5. Busard C, Menting S, van Bezooijen SJ, van den Reek J, Hutten B, Prens E, et al. Erratum to: Optimizing adalimumab treatment in psoriasis with concomitant methotrexate (OPTIMAP): study protocol for a pragmatic, 
single-blinded, investigator-initiated randomized controlled trial. Trials. (2017) 18:113. doi: 10.1186/s13063-017-1848-0

6. Wang J, Dore S. Heme oxygenase-1 exacerbates early brain injury after intracerebral haemorrhage. Brain. (2007) 130:164352. doi: $10.1093 /$ brain/awm095

7. Wang J, Dore S. Heme oxygenase 2 deficiency increases brain swelling and inflammation after intracerebral hemorrhage. Neuroscience. (2008) 155:113341. doi: 10.1016/j.neuroscience.2008.07.004

8. Yang WS, Stockwell BR. Ferroptosis: death by lipid peroxidation. Trends Cell Biol. (2016) 26:165-76. doi: 10.1016/j.tcb.2015.10.014

9. Qian Li, Xiaoning Han, Xi Lan, Yufeng Gao, Chao Jiang, Yufeng Gao, et al. Inhibition of neuronal ferroptosis protects hemorrhagic brain. JCI Insight. (2017) 2:e90777. doi: 10.1172/jci.insight.90777

10. Weiland A, Wang Y, Wu W, Lan X, Han X, Li Q, et al. Ferroptosis and its role in diverse brain diseases. Mol Neurobiol. (2019) 56:488093. doi: 10.1007/s12035-018-1403-3

11. Gao C, Du H, Hua Y, Keep RF, Strahle J, Xi G. Role of red blood cell lysis and iron in hydrocephalus after intraventricular hemorrhage. J Cereb Blood Flow Metab. (2014) 34:1070-5. doi: 10.1038/jcbfm.2014.56

12. Guo F, Hua Y, Wang J, Keep RF, Xi G. Inhibition of carbonic anhydrase reduces brain injury after intracerebral hemorrhage. Transl Stroke Res. (2012) 3:130-7. doi: 10.1007/s12975-011-0106-0

13. Gu Y, Hua Y, Keep RF, Morgenstern LB, Xi G. Deferoxamine reduces intracerebral hematoma-induced iron accumulation and neuronal death in piglets. Stroke. (2009) 40:2241-3. doi: 10.1161/STROKEAHA.108.539536

14. Okauchi M, Hua Y, Keep RF, Morgenstern LB, Schallert T, Xi G. Deferoxamine treatment for intracerebral hemorrhage in aged rats: therapeutic time window and optimal duration. Stroke. (2010) 41:37582. doi: 10.1161/STROKEAHA.109.569830

15. Nakka VP, Prakash-Babu P, Vemuganti R. Crosstalk between endoplasmic reticulum stress, oxidative stress, and autophagy: potential therapeutic targets for acute CNS injuries. Mol Neurobiol. (2016) 53:532-44. doi: 10.1007/s12035-014-9029-6

16. Halliday M, Hughes D, Mallucci GR. Fine-tuning PERK signaling for neuroprotection. J Neurochem. (2017) 142:812-26. doi: 10.1111/jnc.14112

17. Godin JD, Creppe C, Laguesse S, Nguyen L. Emerging roles for the unfolded protein response in the developing nervous system. Trends Neurosci. (2016) 39:394-404. doi: 10.1016/j.tins.2016.04.002

18. Meng C, Zhang J, Dang B, Li H, Shen H, Li X, et al. PERK pathway activation promotes intracerebral hemorrhage induced secondary brain injury by inducing neuronal apoptosis both in vivo and in vitro. Front Neurosci. (2018) 12:111. doi: 10.3389/fnins.2018.00111

19. Zhang J, Zhang P, Meng C, Dang B, Li H, Shen H, et al. The PERK pathway plays a neuroprotective role during the early phase of secondary brain injury induced by experimental intracerebral hemorrhage. Acta Neurochir Suppl. (2020) 127:105-19. doi: 10.1007/978-3-030-04615-6_17

20. Huang L, Woo W, Sherchan P, Khatibi NH, Krafft P, Rolland W, et al. Valproic acid pretreatment reduces brain edema in a rat model of surgical brain injury. Acta Neurochir Suppl. (2016) 121:305-10. doi: 10.1007/978-3-319-18497-5_53

21. You W, Wang Z, Li H, Shen H, Xu X, Jia G, et al. Inhibition of mammalian target of rapamycin attenuates early brain injury through modulating microglial polarization after experimental subarachnoid hemorrhage in rats. J Neurol Sci. (2016) 367:224-31. doi: 10.1016/j.jns.2016.0 6.021

22. Wang Z, Wang Y, Tian X, Shen H, Dou Y, Li H, et al. Transient receptor potential channel $1 / 4$ reduces subarachnoid hemorrhage-induced early brain injury in rats via calcineurin-mediated NMDAR and NFAT dephosphorylation. Sci Rep. (2016) 6:33577. doi: 10.1038/srep33577

23. Ding R, Chen Y, Yang S, Deng X, Fu Z, Feng L, et al. Blood-brain barrier disruption induced by hemoglobin in vivo: Involvement of up-regulation of nitric oxide synthase and peroxynitrite formation. Brain Res. (2014) 1571:2538. doi: 10.1016/j.brainres.2014.04.042

24. Zhang Z, Wu Y, Yuan S, Zhang P, Zhang J, Li H, et al. Glutathione peroxidase 4 participates in secondary brain injury through mediating ferroptosis in a rat model of intracerebral hemorrhage. Brain Res. (2018) 1701:11225. doi: 10.1016/j.brainres.2018.09.012

25. Yang P, Manaenko A, Xu F, Miao L, Wang G, Hu X, et al. Role of PDGF-D and PDGFR- $\beta$ in neuroinflammation in experimental ICH mice model. Exp Neurol. (2016) 283:157-64. doi: 10.1016/j.expneurol.2016.06.010

26. Feng D, Wang B, Wang L, Abraham N, Tao K, Huang L, et al. Pre-ischemia melatonin treatment alleviated acute neuronal injury after ischemic stroke by inhibiting endoplasmic reticulum stress-dependent autophagy via PERK and IRE1 signalings. J Pineal Res. (2017) 62. doi: 10.1111/jpi.12395

27. Roussel BD, Kruppa AJ, Miranda E, Crowther DC, Lomas DA, Marciniak SJ. Endoplasmic reticulum dysfunction in neurological disease. Lancet Neurol. (2013) 12:105-18. doi: 10.1016/S1474-4422(12)70238-7

28. Hammond EJ, Ramsay RE, Villarreal HJ, Wilder BJ. Effects of intracortical injection of blood and blood components on the electrocorticogram. Epilepsia. (1980) 21:3-14. doi: 10.1111/j.1528-1157.1980.tb04039.x

29. Xi G, Keep RF, Hoff JT. Erythrocytes and delayed brain edema formation following intracerebral hemorrhage in rats. J Neurosurg. (1998) 89:9916. doi: 10.3171/jns.1998.89.6.0991

30. Keep RF, Hua Y, Xi G. Intracerebral haemorrhage: mechanisms of injury and therapeutic targets. Lancet Neurol. (2012) 11:72031. doi: 10.1016/S1474-4422(12)70104-7

31. Umbreit J. Methemoglobin-it's not just blue: a concise review. Am J Hematol. (2007) 82:134-44. doi: 10.1002/ajh.20738

32. Imai $H$, Matsuoka $M$, Kumagai $T$, Sakamoto $T$, Koumura $T$. Lipid peroxidation-dependent cell death regulated by GPx4 and ferroptosis. Curr Top Microbiol Immunol. (2017) 403:143-70. doi: 10.1007/82_2016_508

33. Feng-Ping H, Guohua X, Keep RF, Ya H, Andrei N, Hoff JT. Brain edema after experimental intracerebral hemorrhage: role of hemoglobin degradation products. J Neurosurg. (2002) 96:287-93. doi: 10.3171/jns.2002.96.2.0287

34. Keep RF, Zhou N, Xiang J, Andjelkovic AV, Hua Y, Xi G. Vascular disruption and blood-brain barrier dysfunction in intracerebral hemorrhage. Fluids Barriers CNS. (2014) 11:18. doi: 10.1186/2045-8118-11-18

35. Tsivgoulis G, Katsanos AH, Butcher KS, Boviatsis E, Triantafyllou $\mathrm{N}$, Rizos I, et al. Intensive blood pressure reduction in acute intracerebral hemorrhage: a meta-analysis. Neurology. (2014) 83:1523-9. doi: 10.1212/WNL.0000000000000917

Conflict of Interest: The authors declare that the research was conducted in the absence of any commercial or financial relationships that could be construed as a potential conflict of interest.

Copyright (c) $2020 \mathrm{Wu}, \mathrm{Gao}$, Dang and Chen. This is an open-access article distributed under the terms of the Creative Commons Attribution License (CC BY). The use, distribution or reproduction in other forums is permitted, provided the original author(s) and the copyright owner(s) are credited and that the original publication in this journal is cited, in accordance with accepted academic practice. No use, distribution or reproduction is permitted which does not comply with these terms. 